\title{
Cosmic ray nuclei detection in the balloon borne nuclear emulsion gamma ray telescope flight in Australia (GRAINE 2015)
}

\author{
Atsushi Iyono ${ }^{1, a}$, Saya Yamamoto ${ }^{1, b}$, Shigeki Aoki ${ }^{2}$, Toshio Hara ${ }^{2}$, Kenji Kuretsubo ${ }^{2}$, Toshitsugu Marushima ${ }^{2}$, \\ Haruka Matsumoto $^{2}$, Fukashi Mizutani ${ }^{2}$, Keita Ozaki ${ }^{2}$, Emi Shibayama ${ }^{2}$, Atsumu Suzuki ${ }^{2}$, Satoru Takahashi ${ }^{2}$, \\ Yurie Tateishi ${ }^{2}$, Misato Yabu ${ }^{2}$, Kyohei Yamada ${ }^{2}$, Koichi Kodama ${ }^{3}$, Kaname Hamada ${ }^{4}$, Hiroaki Kawahara ${ }^{5}$, Ryosuke \\ Komatani $^{5}$, Masahiro Komatsu ${ }^{5}$, Motoaki Miyanishi ${ }^{5}$, Misaki Morishita ${ }^{5}$, Kunihiro Morishima ${ }^{5}$, Mitsuhiro Nakamura ${ }^{5}$, \\ Naotaka Naganawa ${ }^{5}$, Toshiyuki Nanano ${ }^{5}$, Akira Nishio ${ }^{5}$, Kimio Niwa ${ }^{5}$, Naoto Otsuka ${ }^{5}$, Hiroki Rokujo ${ }^{5}$, Osamu Sato ${ }^{5}$, \\ and Masahiro Yoshimoto ${ }^{5}$

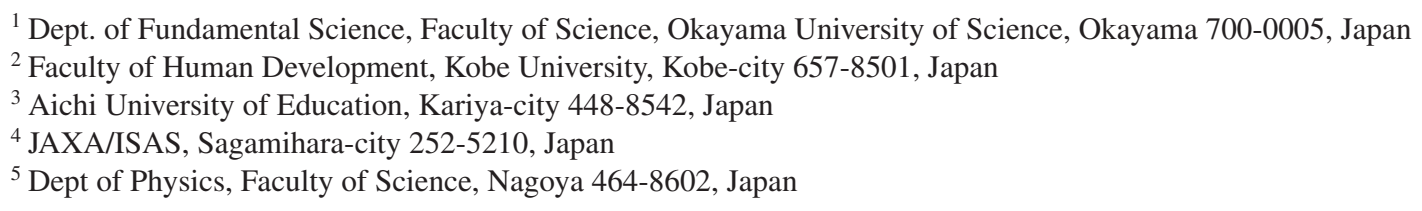

\begin{abstract}
Nuclear emulsion plates for studying elementary particle physics as well as cosmic ray physics are very powerful tracking tools with sub-micron spatial resolutions of charged particle trajectories. Even if gamma rays have to be detected, electron-positron pair tracks can provide precise information to reconstruct their direction and energy with high accuracy. Recent developments of emulsion analysis technology can digitally handle almost all tracks recorded in emulsion plates by using the Hyper Track Selector of the OPERA group at NAGOYA University. On the other hand, the potential of time resolutions have been equipped by emulsion multilayer shifter technology in the GRAINE (Gamma Ray Astro-Imager with Nuclear Emulsion) experiments, the aims of which are to detect cosmic gamma rays such as the Vela pulsar stellar object by precise emulsion tracking analysis and to study cosmic ray particle interactions and chemical compositions. In this paper, we focus on the subject of cosmic ray nuclei detection in the GRAINE balloon flight experiments launched at Alice Springs, Australia in May 2015.
\end{abstract}

\section{Introduction}

The observation of high-energy cosmic gamma rays provides the origins and acceleration mechanism of highenergy cosmic rays in the universe. Recently Fermi-LAT $[1,2]$ reported observations of more than three thousand gamma ray point sources including 30 percent unidentified objects. To explore these sources in detail, a more precise angular resolution has been needed in the gamma ray energy range of $10 \mathrm{MeV}-100 \mathrm{GeV}$.

Nuclear emulsion films are precise tracking detectors which record the charged particle trajectories with less than one micrometer accuracy, three-dimensionally. To detect gamma rays in nuclear emulsion detectors, the leading point of the electron-positron pair created by a gamma-ray has been measured with precise submilliradian angular resolution in emulsion films; the gamma ray arrival direction and momentum can be precisely determined.

On May 12, 2015, a Japan-Australia JAXA collaborative balloon-borne experiment (GRAINE 2015) [3] was successfully launched from the Alice Springs balloonlaunching station to Longreach with a $3780 \mathrm{~cm}^{2}$-aperture telescope for a duration of 14.4 hours, including 11.5 hours of level flight at an altitude of $36.0 \mathrm{~km}$ to

\footnotetext{
a e-mail: iyono@das.ous.ac.jp

b e-mail: S15SM08SY@ous.jp
}

$37.4 \mathrm{~km}\left(4.8 \mathrm{~g} / \mathrm{cm}^{2}\right.$ to $\left.3.9 \mathrm{~g} / \mathrm{cm}^{2}\right)$. The primary purpose of this flight was to confirm the overall system performance and to observe the morphology of well-known and brightest gamma-ray objects such as the Vela pulsar with the gamma-ray emulsion telescope.

The nuclear emulsion films had been exposed to cosmic radiation at this height directly, and we expected comic ray nuclei components including He nuclei and heavier ones. Cosmic ray nuclei provide important information on the origin, propagation and acceleration mechanism of cosmic rays in terms of primary-secondary nuclei ratio. In the GRAINE balloon flight [3], the detection of these cosmic ray nuclei components was examined with image analysis technologies as well as time-stamper systems.

\section{Apparatus}

The structure of the emulsion gamma-ray telescope [3,4] which consists of a converter, time-stamper, calorimeter, and an attitude monitor is shown in Fig. 1. The materials of the converter part make the incident gamma rays convert into electron-positron pairs and these electrons have been registered as three dimensional tracks in the stacks of nuclear emulsion films. The time-stamper, which consists of triple emulsion film shifters as hour, minute 


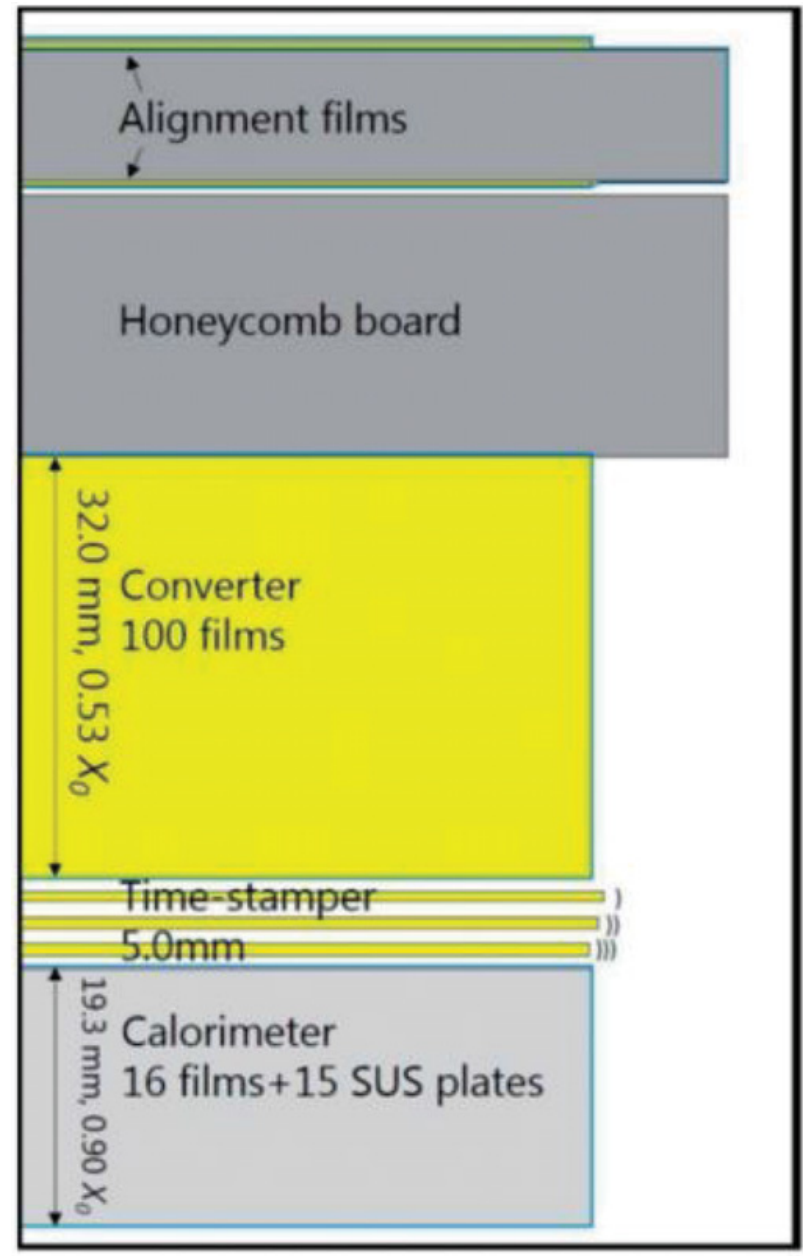

Figure 1. Emulsion telescope structure which consists of the converter, time-stamper and calorimeter.

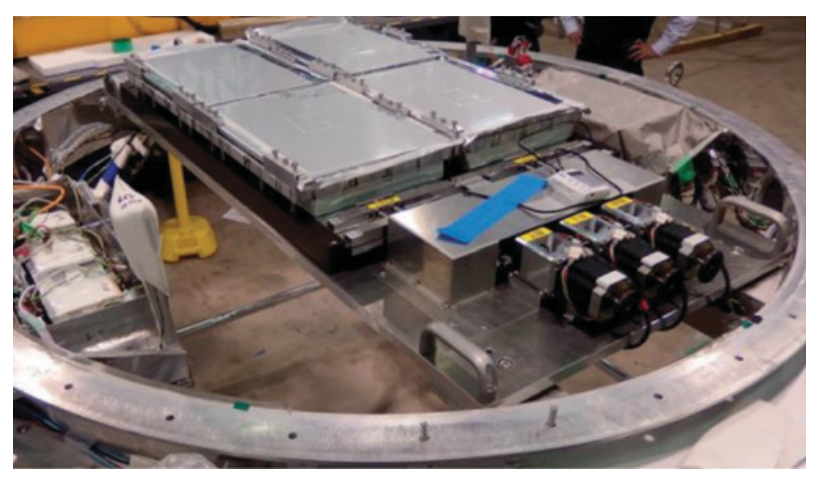

Figure 2. Emulsion chambers in the pressure vessel. Three motors shift emulsion films in the time-stamper part in the three different time period such as hour, minute and second hands of an analog clock.

and second hands of an analog clock shown in Fig. 2, provides an arrival time (or time-stamp) information for all charged tracks. After the recognition and reconstruction of electron pairs, the arrival time and direction as well as the momentum were determined. The arrival time obtained by time-stamping of electron pairs is combined with three star camera images deployed on the balloon gondola shown in Fig. 3, and their arrival directions into the celestial coordinate were determined. These cameras monitored the attitude of gondola to observe the stars positions. The

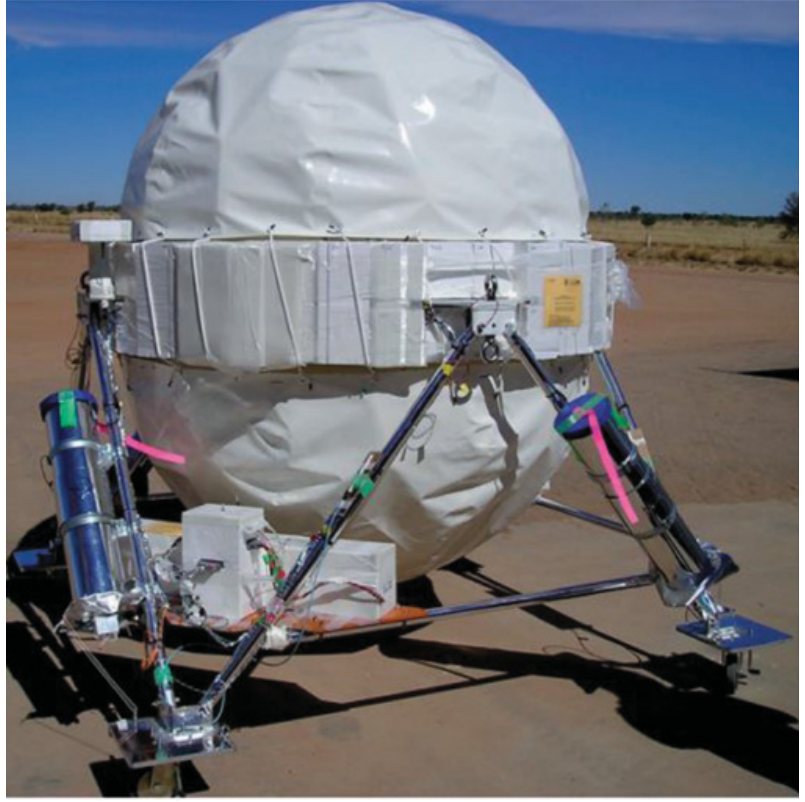

Figure 3. Gondola with thermal shielding. The three star cameras are deployed at the truss structure and view three different directions.

trajectory of charged particles measured in the emulsion films enables an estimation of their momentum by using the multiple Coulomb scattering method up to several hundred $\mathrm{MeV} / \mathrm{c}$.

\section{Emulsion film analysis}

GRAINE2015 emulsion films were recovered after completing the balloon flight at Longreach, and sent to Sydney University for chemical developments within less than one month. The design values of emulsion film thickness are summarized in Table 1. Further chemical treatment for these developed films was handled at Kobe university. The typical thickness of the emulsion part of films after development was very thin (such as $\sim 40 \mu \mathrm{m})$. For further image recognition procedures with high efficiency, we need the original emulsion thickness when exposing them to cosmic radiation. The glycerin swelling process has been carried out for all emulsion films to swell the gelatin part of nuclear emulsion by glycerin infiltration. The recovered thickness was achieved up to $90 \%$ of the nominal thickness.

Table 1. The thickness of emulsion films in each part of chamber.

\begin{tabular}{|c|c|c||c|}
\hline $\begin{array}{c}\text { Part of } \\
\text { detector }\end{array}$ & $\begin{array}{c}\text { Thickness } \\
\text { of emulsion } \\
\text { layer }[\mu \mathrm{m}]\end{array}$ & $\begin{array}{c}\text { Thickness } \\
\text { PS base }[\mu \mathrm{m}]\end{array}$ & $\begin{array}{c}\text { Total thickness } \\
{[\mu \mathrm{m}]}\end{array}$ \\
\hline \hline Converter & 70 & 180 & 320 \\
Time-stamper & 42 & 180 & 264 \\
& 40 & 40 & 120 \\
Calorimeter & 45 & 180 & 270 \\
\hline
\end{tabular}

PS: polystyrene base film.

In the emulsion scanning process, GRAINE has used the high performance and high speed track selector HTS (Hyper Track Selector shown in Fig. 4) developed 


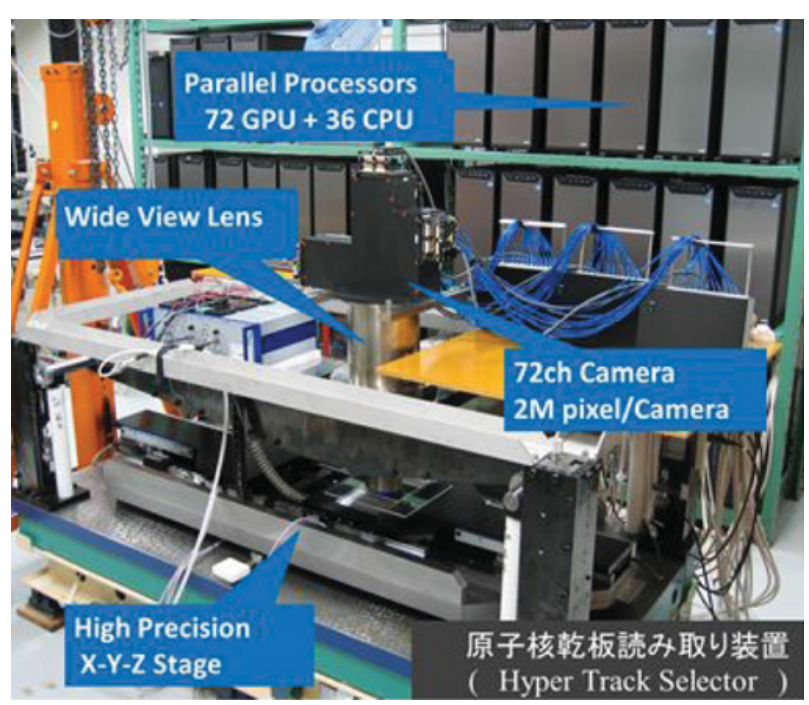

Figure 4. Hyper Track Selector (HTS) implemented at the Fundamental particle laboratory in Nagoya University.

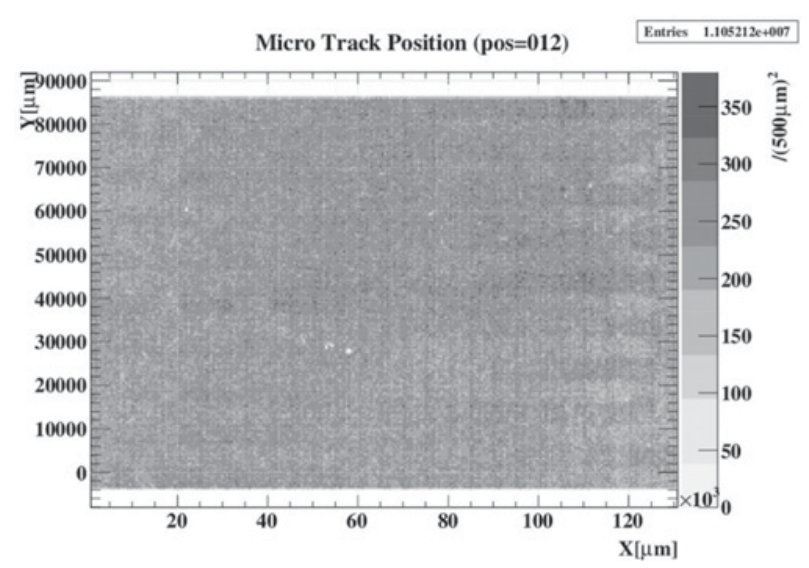

Figure 5. HTS enables general scanning of emulsion films extended to $8 \mathrm{~cm} \times 12 \mathrm{~cm}$ area within $\tan \theta \leq 2$. The map shows the track density per $500 \times 500 \mu \mathrm{m}^{2}$.

and implemented at the Fundamental Particle Laboratory (F-lab.) [5,6] in Nagoya University. HTS achieves over $5000 \mathrm{~cm}^{2}$ per hour scanning speed. Figure 5 is the identified track density map in one HTS scanning command. This capability provides the general scan method for the emulsion analysis with wide zenith angle allowance for cosmic ray nuclei studies. To recognize charged tracks in the emulsion films, HTS sliced both upper and bottom emulsion layers into 32 digitized images in GPU memory space. The GPU pipeline logically shifted them in two dimensional directions and summed the image overlapping along the specified zenith angle and orientation angle. The Pulse Height (PH) and Pulse Height Volume (PHV) were defined as the number of overlapped pixels and the total number of $\mathrm{PH}$ values above threshold values (typically 7 or 8), respectively. The HTS scanning algorithm is shown in Fig. 6. The nominal allowance of zenith angle was extended up to 63 degrees $(\tan \theta \leq 2.0)$ in the GRAINE2015 analysis and the track recognition efficiency became more than 95\%. The extension of angular allowances and quick scan capability in HTS made the cosmic ray nuclei analysis in GRAINE experiments possible.
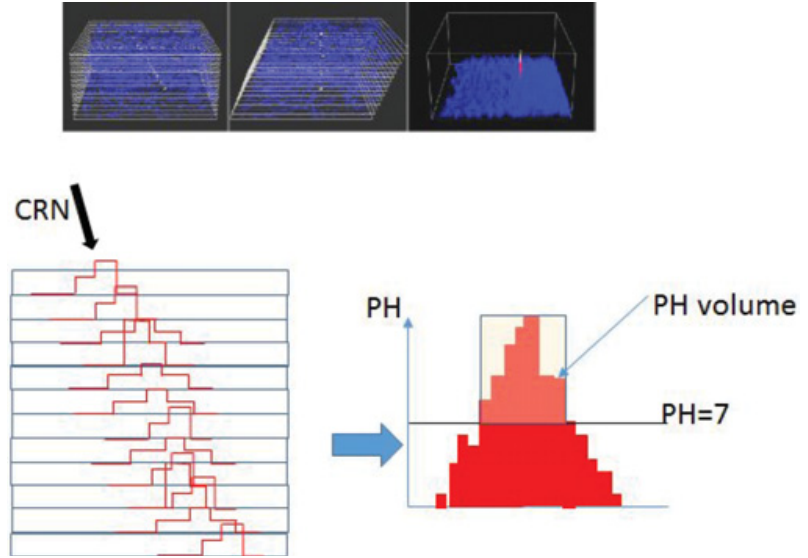

Figure 6. Hyper Track Selector (HTS) logical algorithm to search for tracks and definitions. The symbol:CRN represents incident charged particle to emulsion layer and $\mathrm{PH}$ and $\mathrm{PH}$ volume are the number of overlapped pixels and summed pixel number above $\mathrm{PH}$ greater than 7, respectively.

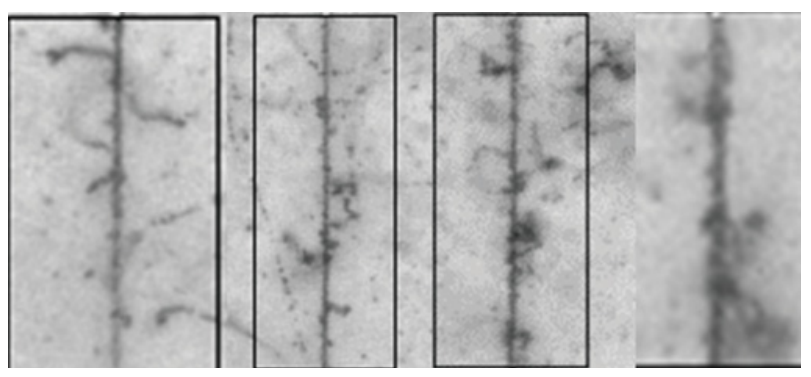

Figure 7. 4 example raw images for high PHV tracks. Images are processed by applying dark peak fold filter and accumulated along the track directions.

\section{Pulse height volume (PHV) analysis}

The pulse height volume defined in Sect. 3 could provide the ionization information of each track's $(Z / \beta)$. We selected 50 tracks for both $\tan \theta<0.6$ and $\tan \theta>1.0$ with the highest PHV signals and obtained their raw images by using the HTS manual mode operations shown in Fig. 7. Most of them were associated with knock-on electrons ( $\delta$ rays), which indicated the incidence of cosmic ray heavier nuclei. Because the number of $\delta$ rays were very limited, we did not go into detail for its relation to PHV data at present.

We analyzed about $10^{6}$ tracks in each emulsion film and followed them among 100 emulsion film stacks. The tracking efficiencies between three films were typically 95\%. We examined the PHV distribution in one emulsion film. The PHV data for single charged tracks depend on their incident zenith angles because all grains overlapped for the case of $\tan \theta=0$ and sparsely overlapped for horizontal tracks. The PHV distributions for each zenith angle group are shown in Fig. 8. The mode values of PHV distributions decreased as their zenith angles increased, due to the grain separation of single charged tracks. On the other hand, the PHV highest values became larger as the zenith angle increased, because the horizontal tracks blurred some multiple sliced images. We demonstrated the normalized PHV distribution by fixing the mode value equal to 50, the second peak PHV value above 100 and 


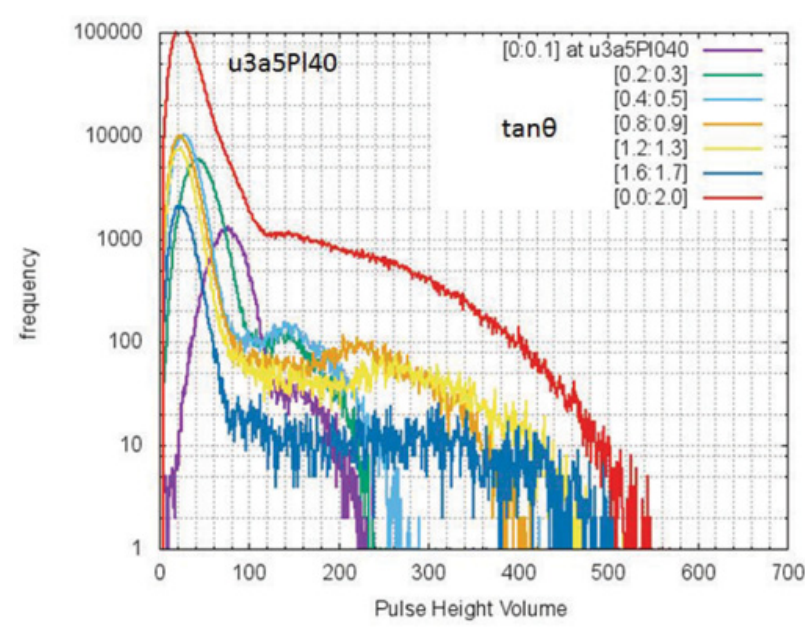

Figure 8. PHV distribution at one emulsion layer. The horizontal axis represents PHV. Each color represents a zenith angle group.

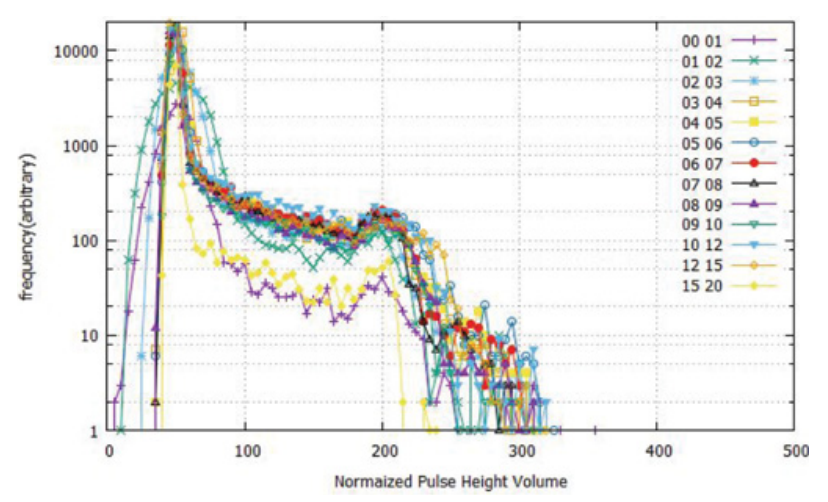

Figure 9. Normalized PHV distribution at emulsion layer. Horizontal axis represents nomalized PHV. Each color represents a zenith angle $(\times 10)$ group.

minimum PHV values. The obtained nomalized PHV distributions for several zenith angle groups are shown in Fig. 9. Around the PHV mode, these distributions scattered with zenith angle. This could come from the grain overlapping effects. But nomalized PHV distributions were well described with one shape above $P H V \geq 100$. This could provide some universality of the relation of PHV to zenith angle. In these distributions, we clearly obtained a second peak for each zenith angle group. The zenith angle dependency of the nomalization parameters (minimum, mode and maximum PHV values) were plotted as a function of zenith angles in Fig. 10. We have still not concluded these feature of PHV zenith angle dependency to identify this second PHV peak as cosmic ray nuclei components so far.

\section{Pulse height volume distributions at sea level and flight level}

The GRAINE project has developed and utilized emulsion film shifters in order to determine charged track arrival timing described above. This system enables us to distinguish between the accumulation of background muon tracks at sea level and cosmic rays in flight by using track arrival timing. We set up emulsion shifters at special coordinates such as an origin when waiting for

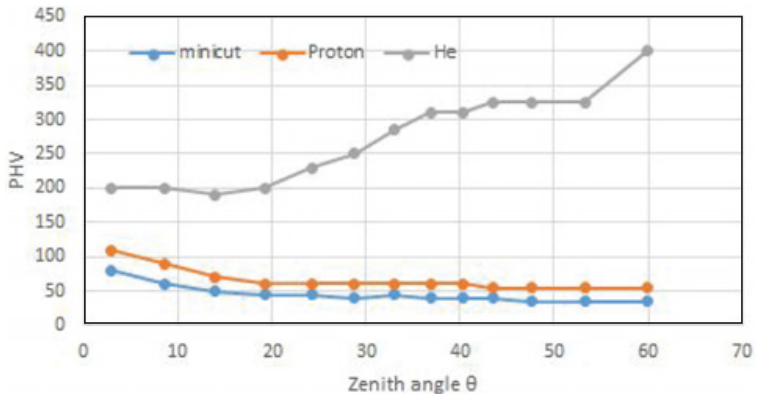

Figure 10. The parameters of the normalized PHV distribution dependence on zenith angle. The minimum, mode and maximum $\mathrm{PHV}$ values are indicated by minicut, Proton and He respectively.

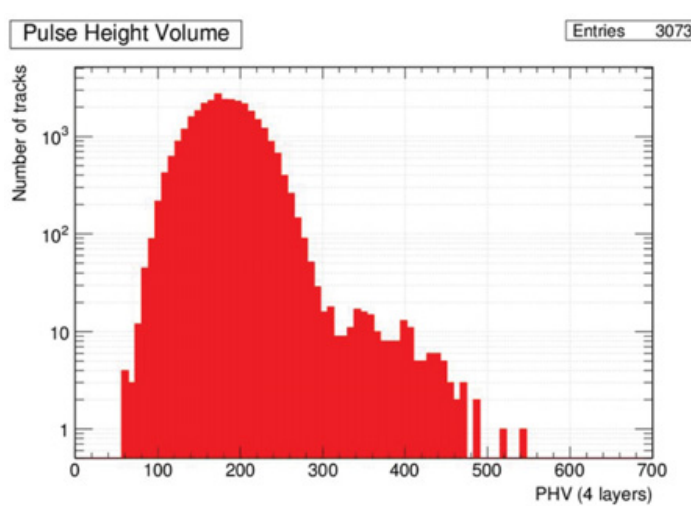

Figure 11. PHV distributions at ground level.

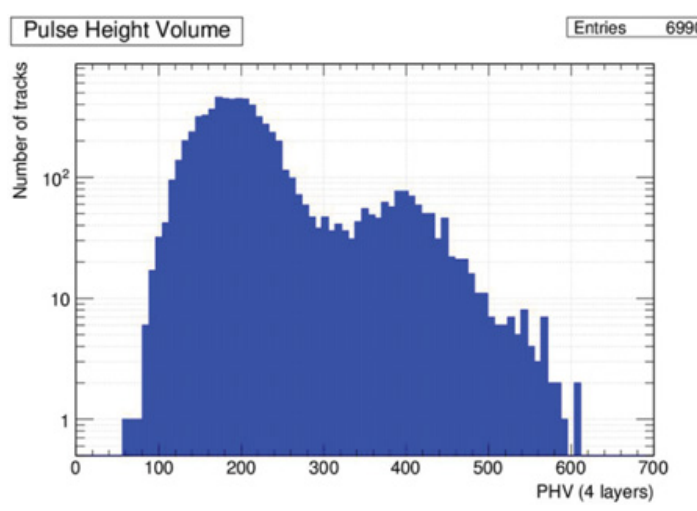

Figure 12. PHV distributions at ground level.

the balloon launch. In the flight, three shifter stages moved in each proper period like hour, minute and second hands of an analog clock. The PHV distribution of the tracks which penetrated the chambers at sea level is shown in Fig. 11. The horizontal axis represents the PHV summation between 4 emulsion layers, and then PHV values were twice as large as the previous ones. In this distribution, the mode PHV $(\sim 180)$ came mainly from single charged particles such as EAS muons. Above PHV equal to 300 , the second peak of PHV distribution was unseen and the maximum PHV does not reach 500. On the other hand, the PHV distribution obtained in the balloon flight shown in Fig. 12 differed from the previous one especially above PHV $=300$. We have clearly seen the second peak at around PHV $=400$ and the maximum values extend to $\mathrm{PHV}=600$. This difference 
came from cosmic ray Helium and heavier nuclei contributions in the high PHV range. This result proved the potential of cosmic ray nuclei measurement in GRAINE projects.

\section{Conclusion}

The GRAINE projects successfully completed a balloon flight at Alice Springs, Australia of 14 hours duration. The overall system worked well within the design accuracies. The emulsion film handling before and after the balloon flight was controlled very well in both Australia and Japan. The swelling process before HTS image recognition process has been established in order to control the emulsion layer thickness suitable for HTS scanning efficiency.

The pulse height volume obtained by the HTS track recognition process was able to be used for cosmic ray nuclei identification. The PHV distributions provided cosmic ray nuclei composition information. Finally, the time-stamper system can distinguish the ground level EAS muons and flight-components of direct cosmic rays, by using the arrival timing of cosmic rays.

The qualitative characteristics of pulse height volume (PHV) were successfully derived from these analyses so far. But we have to understand the quantitative estimation of PHV dependencies on the incident zenith angles. We still continue data analyses of track data in the GRAINE2015 balloon flight.

\section{References}

[1] F. Acero et al., Astrophys. J. 218, 23, (2015)

[2] C. Gene et al., Astrophys. J.L. L13, 797 (2014)

[3] S. Takahashi et al., Prog. Theor. Exp. Phys. 073F01, 12 (2016)

[4] H. Rokujo et al., Proc. 34th Int. Cosmic Ray Conference The Hague, PoS(ICRC2015)1021, (2015)

[5] K. Niwa et al., Proc. Int. Cosmic Ray Symp. High Energy Phenomena Tokyo, 149 (1974)

[6] M. Yoshimoto, Master's thesis, Nagoya University, Japan (2015) (in Japanese) 